\title{
Video Article \\ Real-time Observation of the DNA Strand Exchange Reaction Mediated by Rad51
}

\author{
Kentaro Ito ${ }^{1}$, Bilge Argunhan ${ }^{1}$, Hideo Tsubouchi ${ }^{1}$, Hiroshi Iwasaki ${ }^{1}$ \\ ${ }^{1}$ Cell Biology Center, Institute of Innovative Research, Tokyo Institute of Technology
}

Correspondence to: Bilge Argunhan at bargunhan@bio.titech.ac.jp, Hiroshi Iwasaki at hiwasaki@bio.titech.ac.jp

URL: https://www.jove.com/video/59073

DOI: doi:10.3791/59073

Keywords: Biochemistry, Issue 144, Homologous recombination, DNA strand exchange reaction, Rad51, Swi5-Sfr1, real-time observation, fluorescence resonance energy transfer (FRET)

Date Published: 2/13/2019

Citation: Ito, K., Argunhan, B., Tsubouchi, H., Iwasaki, H. Real-time Observation of the DNA Strand Exchange Reaction Mediated by Rad51. J. Vis. Exp. (144), e59073, doi:10.3791/59073 (2019).

\section{Abstract}

The DNA strand exchange reaction mediated by Rad51 is a critical step of homologous recombination. In this reaction, Rad51 forms a nucleoprotein filament on single-stranded DNA (ssDNA) and captures double-stranded DNA (dsDNA) non-specifically to interrogate it for a homologous sequence. After encountering homology, Rad51 catalyzes DNA strand exchange to mediate pairing of the ssDNA with the complementary strand of the dsDNA. This reaction is highly regulated by numerous accessary proteins in vivo. Although conventional biochemical assays have been successfully employed to examine the role of such accessory protein in vitro, kinetic analysis of intermediate formation and its progression into a final product has proven challenging due to the unstable and transient nature of the reaction intermediates. To observe these reaction steps directly in solution, fluorescence resonance energy transfer (FRET)-based real-time observation systems of this reaction were established. Kinetic analysis of real-time observations shows that the DNA strand exchange reaction mediated by Rad51 obeys a three-step reaction model involving the formation of a three-strand DNA intermediate, maturation of this intermediate, and the release of ssDNA from the mature intermediate. The Swi5-Sfr1 complex, an accessary protein conserved in eukaryotes, strongly enhances the second and third steps of this reaction. The FRET-based assays presented here enable us to uncover the molecular mechanisms through which recombination accessary proteins stimulate the DNA strand exchange activity of Rad51. The primary goal of this protocol is to enhance the repertoire of techniques available to researchers in the field of homologous recombination, particularly those working with proteins from species other than Schizosaccharomyces pombe, so that the evolutionary conservation of the findings presented herein can be determined.

\section{Video Link}

The video component of this article can be found at https://www.jove.com/video/59073/

\section{Introduction}

Homologous recombination (HR) facilitates the shuffling of genetic information between two different DNA molecules. HR is essential for two fundamental biological phenomena: the generation of genetic diversity during gametogenesis ${ }^{1}$ and the repair of DNA double-strand breaks $(\mathrm{DSBs})^{2}$ during mitosis. DSBs are the most severe form of DNA damage and constitute a breakage in the chromosome. Incorrect repair of DSBs can cause extensive chromosomal rearrangements and genomic instability, which are both hallmarks of cancer ${ }^{3}$.

The DNA strand exchange reaction is the central phase of HR. The Rad51 protein, which is a member of the highly conserved RecA-type family of recombinases, is the key protein that catalyzes this reaction in eukaryotes ${ }^{4,5}$. In this reaction, Rad51 binds to single-stranded DNA (ssDNA) generated by nucleolytic processing of the DSB end and forms a helical nucleoprotein complex termed the presynaptic filament. This filament catches intact double-stranded DNA (dsDNA) nonspecifically to search for a homologous sequence. When the filament finds a homologous sequence, a reaction intermediate containing three-stranded DNA is formed and the Rad51 filament mediates strand exchange within this structure ${ }^{6,7,8}$. To accomplish this reaction efficiently, Rad51 requires several kinds of accessory proteins such as BRCA1 and BRCA2, products of breast cancer susceptibility genes ${ }^{9,10}$.

Understanding how accessory factors regulate Rad51 is an integral step in uncovering the causes of genomic instability during tumorigenesis Although much research is concerned with the effects of these factors on presynaptic filament formation and stability ${ }^{11,12,13,14,15,16}$, the contribution of these factors to formation of the three-strand intermediate and its processing into the final product is still unclear. Observing these reaction steps through conventional biochemical experiments is very difficult because the three-strand intermediate is unstable and prone to collapse by common experimental manipulations such as deproteinization of samples or electrophoresis.

To overcome this problem, we adapted two previously developed real-time observation systems of the DNA strand exchange reaction using fluorescence resonance energy transfer (FRET): the DNA strand pairing and DNA strand displacement assays ${ }^{17,18}$ (Figure 1). In the DNA strand pairing assay, Rad51 forms a presynaptic filament with fluorescein amidite (FAM)-labeled SsDNA and then homologous carboxy-x-rhodamine (ROX)-labeled dsDNA is added to initiate the strand exchange reaction. When the filament catches the ROX-labeled dsDNA and forms the threestrand intermediate, the two fluorophores come into close proximity and fluorescence emission of FAM is quenched by ROX (Figure 1A). In the 
DNA strand displacement assay, a presynaptic filament formed on unlabeled ssDNA is incubated with FAM and ROX double-labeled dsDNA. When strand exchange is completed and the FAM labeled ssDNA is released from the three-strand intermediate, the emission of FAM increases because FAM is no longer in close proximity to ROX (Figure 1B). These assays enable us to observe the formation of three-strand intermediates and their processing into final products in real-time without any disturbances to the reaction.

Using this real-time observation system, we found that the DNA strand exchange reaction mediated by Rad51 proceeds in three-steps including the formation of the first reaction intermediate (C1), transitioning of the first intermediate into a second intermediate (C2), and release of ssDNA from $\mathrm{C} 2^{19}$. We also found that fission yeast (S. pombe) Swi5-Sfr1, which is an evolutionarily conserved Rad51 accessory protein complex ${ }^{13,16,20,21,22}$, stimulates the C1-C2 transitioning and release of ssDNA from C2 in a manner that is dependent on ATP-hydrolysis by Rad51 $1^{19}$.

Whether these findings are evolutionarily conserved remains unknown. This protocol is provided with the hope that researchers in the field of $\mathrm{HR}$, especially those working with proteins from organisms other than S. pombe, may apply these techniques to determine the extent to which the molecular mechanism of Rad51-driven strand exchange is conserved. Furthermore, these techniques have proven highly successful in determining the role of $S$. pombe Swi5-Sfr1. Thus, it is a rational prediction that these techniques will be invaluable in uncovering the precise roles of other HR accessory factors.

\section{Preparation of Proteins and DNA Substrates}

1. Purify S. pombe Rad51 and Swi5-Sfr1 proteins to homogeneity (judged by Coomassie staining), as previously reported ${ }^{13,21}$.

2. Prepare oligonucleotide DNA substrates listed in Table $1^{18}$.

NOTE: The oligonucleotides were purchased (see Table of Materials) and synthesized at HPLC grade. For the DNA strand pairing reaction, oligonucleotides $16 \mathrm{FA}(-), 16 \mathrm{~A}(-) \_40 \mathrm{bp}$ and $16 \mathrm{AR}(+) \_40 \mathrm{bp}$ are required. For the DNA displacement assay, oligonucleotides 16A(-), 16FA(-)_40bp and 16AR(+)_40bp are required (Figure 1 and Table 1). All DNA concentrations in this protocol refer to fragment concentrations as opposed to nucleotide concentrations.

3. To form donor dsDNA, mix equimolar amounts of complementary strands in a thin-walled PCR tube with annealing buffer (10 mM Tris- $\mathrm{HCl} \mathrm{pH}$ $7.5,100 \mathrm{mM} \mathrm{NaCl}, 10 \mathrm{mM} \mathrm{MgCl}_{2}$ ), ensuring a total volume greater than $20 \mu \mathrm{L}$. Perform this mixing on a prechilled metal rack on ice (between $2{ }^{\circ} \mathrm{C}$ and $4{ }^{\circ} \mathrm{C}$ ).

NOTE: The combination of oligonucleotides for the pairing assay are $16 \mathrm{~A}(-) \_40 \mathrm{bp}$ and $16 \mathrm{AR}(+) \_40 \mathrm{bp}$. For the displacement assay, anneal oligonucleotides 16FA(-) 40bp and 16AR(+) 40bp.

4. Heat the annealing mixture at $90^{\circ} \mathrm{C}$ for $5 \mathrm{~min}$ and cool down over $3 \mathrm{~h}$ to $30^{\circ} \mathrm{C}$ using a PCR machine. Store the annealed DNA at $-20{ }^{\circ} \mathrm{C}$

\section{DNA Strand Pairing and Displacement Assays}

1. Perform DNA strand pairing assay.

1. Prepare $1.6 \mathrm{~mL}$ of reaction buffer $\mathrm{A}(30 \mathrm{mM}$ HEPES-KOH pH 7.5, $1 \mathrm{mM}$ dithiothreitol [DTT], $15 \mathrm{mM} \mathrm{MgCl}, 0.25 \mathrm{mM} \mathrm{ATP}, 0.1 \mathrm{mg} /$ $\mathrm{mL}$ bovine serum albumin [BSA], and $0.0075 \%$ polyoxyethylenesorbitan monolaurate) containing $36 \mathrm{nM} 16 \mathrm{FA}(-)$ in a $2.0 \mathrm{~mL}$ microcentrifuge plastic tube (polypropylene) and pre-incubate it at $37^{\circ} \mathrm{C}$ for $5 \mathrm{~min}$.

2. To form Rad51-ssDNA filaments, add Rad51 protein to a final concentration of $1.5 \mu \mathrm{M}$ in the pre-incubated reaction buffer and incubate it at $37^{\circ} \mathrm{C}$ for $5 \mathrm{~min}$.

3. Add Swi5-Sfr1 protein to the mixture to a final concentration of $0.15 \mu \mathrm{M}$ and incubate it at $37^{\circ} \mathrm{C}$ for a further $5 \mathrm{~min}$.

4. Take $1.5 \mathrm{~mL}$ of the mixture and transfer it into a $1.0 \times 1.0 \mathrm{~cm}$ quartz cuvette containing a magnetic stirrer and set the cuvette into a spectrofluorometer. Configure the peltier temperature controller of the spectrophotometer to $37^{\circ} \mathrm{C}$ and set the magnetic stirrer to 450 rpm to ensure rapid mixing of the injected sample.

5. Start measuring the fluorescence emission of FAM at $525 \mathrm{~nm}$ (bandwidth: $20 \mathrm{~nm}$ ) upon excitation at $493 \mathrm{~nm}$ (bandwidth: $1 \mathrm{~nm}$ ). Collect data every second.

6. After starting the measurement for $100 \mathrm{~s}$, inject ROX-labeled donor dsDNA to a final concentration of $36 \mathrm{nM}$ into the mixture using a syringe and measure the change in emission at $1 \mathrm{~s}$ intervals for a further $30 \mathrm{~min}$.

2. Perform DNA strand displacement assay.

1. Prepare $1.6 \mathrm{~mL}$ of reaction buffer $\mathrm{A}$ containing $36 \mathrm{nM} 16 \mathrm{~A}(-)$ in a $2.0 \mathrm{~mL}$ micro-centrifuge plastic tube and pre-incubate it at $37{ }^{\circ} \mathrm{C}$ for 5 $\min$.

2. Form Rad51-ssDNA filaments in the presence of Swi5-Sfr1 at $37^{\circ} \mathrm{C}$, as described in steps 2.1.2. and 2.1.3.

3. Take $1.5 \mathrm{~mL}$ of the mixture and transfer it into the quartz cuvette containing a magnetic stirrer and set the cuvette into the spectrophotometer, as described in step 2.1.4.

4. Start measuring the fluorescence emission and after $100 \mathrm{~s}$, inject FAM- and ROX-labeled donor dsDNA as described in step 2.1.6. Measure the change in fluorescence emission at $1 \mathrm{~s}$ intervals for a further $30 \mathrm{~min}$.

\section{Analyzing Experimental Data from the Pairing and Displacement Assays}

1. Estimate maximum FRET efficiency.

1. Prepare 16FA(-) annealed with $16 \mathrm{AR}(+) \_40 \mathrm{bp}$ and $16 \mathrm{FA}(-) \_40 \mathrm{bp}$ annealed with $16 \mathrm{AR}(+) \_40 \mathrm{bp}$ using the same procedure described in steps 1.3 and 1.4 .

2. Prepare $130 \mu \mathrm{L}$ of reaction buffer $\mathrm{A}$ containing $36 \mathrm{nM}$ of either $16 \mathrm{FA}(-), 16 \mathrm{FA}(-)$ annealed with $16 \mathrm{AR}(+) \_40 \mathrm{bp}, 16 \mathrm{FA}(-) \_40 \mathrm{bp}$ annealed with 16AR(+)_40bp or $16 \mathrm{FA}(-) \_40 \mathrm{bp}$ in a $0.2 \times 1.0 \mathrm{~cm}$ quartz cuvette. 
3. Set the cuvette into the spectrofluorometer and incubate at $37^{\circ} \mathrm{C}$ for $5 \mathrm{~min}$.

4. Measure fluorescence spectra from 500 to $600 \mathrm{~nm}$ upon excitation at $493 \mathrm{~nm}$.

5. To test the effect of Rad51 on the emission of FAM and quenching of FAM by ROX, add Rad51 to a final concentration of $1.5 \mu \mathrm{M}$ to the mixture and incubate at $37^{\circ} \mathrm{C}$ for $5 \mathrm{~min}$.

6. Measure fluorescence spectra from 500 to $600 \mathrm{~nm}$ upon excitation at $493 \mathrm{~nm}$.

7. Calculate maximum $F R E T$ efficiency $\left(E_{\text {maximum }}\right)$ using the equation described below:

$\mathrm{E}_{\text {maximum }}=($ Fluorescence intensity at $525 \mathrm{~nm}$ of dsDNA labeled with both FAM and ROX) / (Fluorescence intensity at $525 \mathrm{~nm}$ of FAM labeled ssDNA)

2. Analyze experimental data from the displacement assay.

1. To convert the change in fluorescence observed in the displacement assay to the change in the amount of final product, normalize raw experimental data obtained from this assay using the equation described below, where $F_{r a w}$ is fluorescence intensity from raw data and $F_{\text {normalized }}$ is the change in fluorescence calculated by the following equation.

$F_{\text {normalized }}=\left(\left[F_{\text {raw }}\right.\right.$ at time $\left.x\right]-\left[F_{\text {raw }}\right.$ at time 0$\left.]\right) /\left(\left(\left[F_{\text {raw }}\right.\right.\right.$ at time 0$\left.] / E_{\text {maximum }}\right)-\left[F_{\text {raw }}\right.$ at time 0$\left.]\right)$

$F_{\text {raw }}$ at time 0 is the average fluorescence monitored for the first $5 \mathrm{~s}$ after the dead time (i.e., the time required for mixing after an injectant is introduced into the cuvette).

2. To exclude the effects of photobleaching and spontaneous displacement, subtract $F_{\text {normalized }}$ without protein from $F_{\text {normalized }}$ of the sample to obtain $\mathrm{F}_{\mathrm{D}}$, which is the change in the amount of final product in this assay. $F_{D}=\left[F_{\text {normalized }}\right.$ of sample $]-\left[F_{\text {normalized }}\right.$ without protein $]$

3. Analyze experimental data from the pairing assay

1. Normalize raw experimental data obtained from the pairing assay using the equation described below, where $F_{\text {raw }}$ is fluorescence intensity from raw data and $F_{\text {normalized }}$ is the change in fluorescence calculated by the following equation.

$\mathrm{F}_{\text {normalized }}=\left(\mathrm{F}_{\text {raw }}\right.$ at time $\left.\mathrm{x}\right) /\left(\mathrm{F}_{\text {raw }}\right.$ at time 0$)$

$F_{\text {raw }}$ at time 0 is the average fluorescence monitored for the last $20 \mathrm{~s}$ before initiating the reaction by injecting the dsDNA substrate.

2. To convert change in fluorescence into change in amount of substrate and exclude the effects of photobleaching and spontaneous pairing, normalize $F_{\text {normalized }}$ of sample using the equation described below, where $F_{p}$ is the change in amount of substrate in this assay. $F_{P}=1-\left(\left(\left[F_{\text {normalized }}\right.\right.\right.$ without protein $]-\left[F_{\text {normalized }}\right.$ of sample $\left.\left.]\right) /\left[1-E_{\text {maximum }}\right]\right)$

3. To examine the reaction kinetics of DNA strand exchange, perform non-linear last-square regression analysis of the pairing reaction using the analysis program ${ }^{23}$ (see Table of Materials).

1. Prepare a file in .txt format containing time course data of $F_{p}$.

2. Start the program and paste the script in the Supplemental Code File into a window of the program:

3. Start non-linear last-square regression analysis. The results of this analysis will be displayed in the same window.

\section{Representative Results}

In order to effectively analyze the experimental data from the pairing and displacement assays, it is necessary to define how a change in fluorescence emission of FAM corresponds to a conversion of DNA substrates into products. To achieve this, the relevant range of fluorescence intensity must be determined. For the pairing assay, the fluorescence emission of $16 \mathrm{FA}(-)$, which corresponds to the ssDNA substrate, is compared with the emission of 16FA(-) annealed with $16 \mathrm{AR}(+)$ - $40 \mathrm{bp}$, which corresponds to the final products of this reaction (Figure 2A). This equates to the maximum FRET efficiency and hence the maximum reduction in fluorescence intensity that would be expected if all ssDNA substrate was converted into the dsDNA product. For the displacement assay, the emission of 16FA(-)_40bp annealed with 16AR(+)_40bp, which corresponds to the substrate, is compared with the emission of 16FA(-)_40bp, which corresponds to the final product (Figure 2B). In this case, the maximum increase in fluorescence intensity of FAM conveys a scenario in which all of the dsDNA substrate is converted into ssDNA product. S. pombe Rad51 did not affect fluorescence emission of FAM or quenching efficiency of FAM by ROX in both assays (Figure 2). The maximum FRET efficiency should be re-measured with each new preparation of oligonucleotides as it is dependent on the labeling efficiency of oligonucleotides.

Representative data of DNA strand pairing and displacement reactions are shown in Figure 3. The effects of spontaneous reactions between substrate DNAs and photobleaching were small in both assays, as revealed by the negligible changes seen in the emission of FAM without Rad51 compared to the substantial changes seen with Rad51 (Figure 3A and Figure 3B). Based on the data shown in Figure 3A or Figure 3B, the change in fluorescence was converted into the change in amount of substrate $\left(F_{P}\right)$ or final product $\left(F_{D}\right)$, respectively, using the equations described in steps 3.2.2 or 3.3.2 (Figure 3C and Figure 3D).

The pairing reaction was simulated using a sequential three-step reaction model, consisting of the formation of the first three-strand intermediate (C1), transitioning of the first intermediate into the second intermediate (C1 to $C 2)$, and the release of ssDNA from the second intermediate to form the two products $(D+E)$ (Figure 3E). To test whether the simulation using a sequential three-step reaction model fit the experimental data, residuals between experimental data of the DNA strand pairing assay and a theoretical curve obtained by the simulation were calculated (Figure 3F). In addition, residuals between the pairing reaction and a theoretical curve generated using a sequential two-step reaction model were also calculated (Figure 3G). The residuals for the pairing reaction and the two-step model show a systematic deviation in the early stage, whereas the residuals for the pairing reaction and the three-step model do not show such a deviation. This indicates that the three-step model is a better fit than the two-step model for simulating the pairing reaction. 
To test whether the three-step model is consistent with the displacement reaction that detects the late step of DNA strand exchange, we generated a theoretical curve of the displacement reaction using the kinetic parameters obtained from simulation of the pairing reaction shown in Figure 3C and compared it with the experimental data of the displacement reaction shown in Figure 3D (Figure 3H). The theoretical curve fit the experimental data of the displacement assay. From these results, we conclude that simulation using the three-step model is able to reasonably evaluate the DNA strand exchange reaction mediated by Rad51.

Representative data of the DNA strand pairing reaction containing Rad51 and the Swi5-Sfr1 complex, an accessary protein of Rad51, are shown in Figure 4A. The Swi5-Sfr1 complex strongly stimulated the pairing activity of Rad51. As was seen in the absence of Swi5-Sfr1, the pairing reaction better fit the three-step model than the two-step model in the presence of Swi5-Sfr1 (Figure 4B). Through simulation of the reaction using the three-step model, reaction equilibrium constants of each reaction step with or without Swi5-Sfr1 were calculated. The reaction equilibrium constants indicated that the Swi5-Sfr1 complex does not stimulate the first reaction step (Figure 4C, panel a), in which a three-strand intermediate is formed, but strongly stimulates C1-C2 transitioning (Figure 4C, panel b) and the release of ssDNA from the C2 intermediate (Figure 4C, panel c).

A

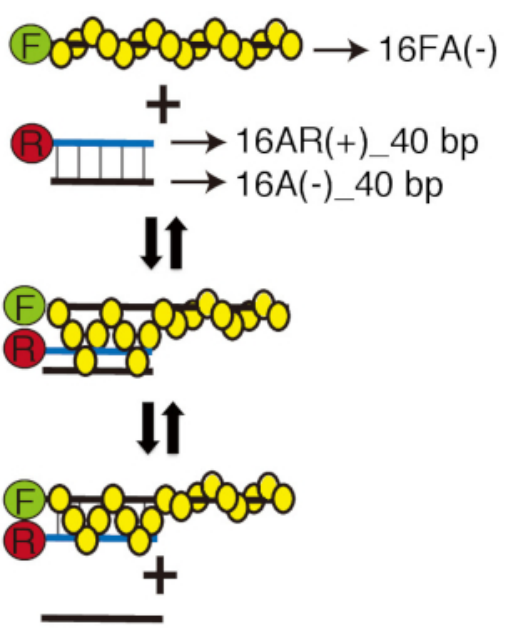

B

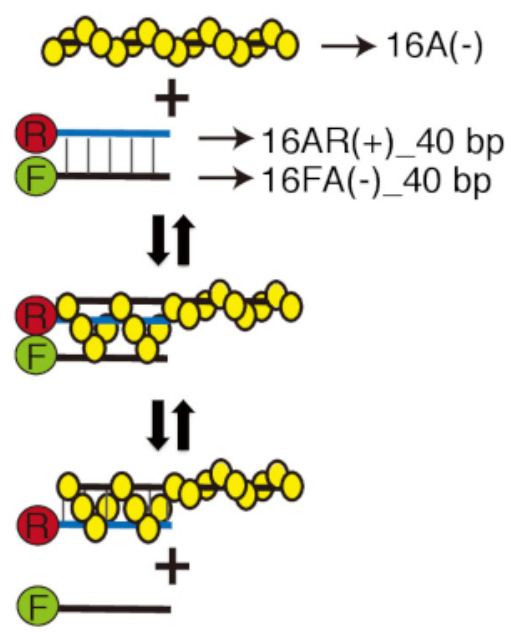

Figure 1: Experimental design of DNA strand pairing and displacement assays. Schematics of DNA strand pairing (A) and displacement (B) assays. Yellow circles represent Rad51 monomers. Green circles containing "F" and red circles containing "R" represent fluorescein amidite (FAM) and carboxy-X-rhodamine (ROX), respectively. Black DNA strands are identical in sequence and complementary to blue DNA strands. Thin black lines with arrowheads point to the name of each oligonucleotide, as depicted in Table 1 . This figure has been adapted from Ito et al. ${ }^{19}$ and modified. Please click here to view a larger version of this figure.

A

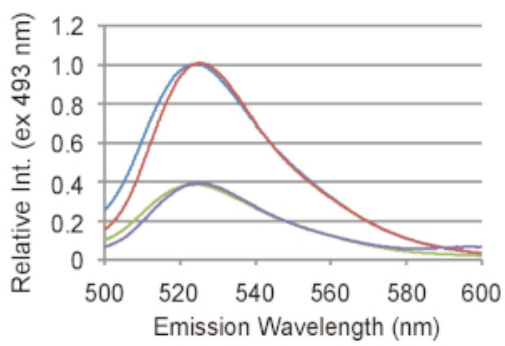

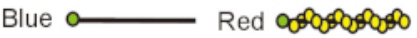

$$
\begin{gathered}
\text { Green } 8=\text { (16FA }(-)) \\
(16 \mathrm{FA}(-) \\
\left.-16 \mathrm{AR}(+) \_40 \mathrm{bp}\right)
\end{gathered}
$$

$\mathrm{B}$

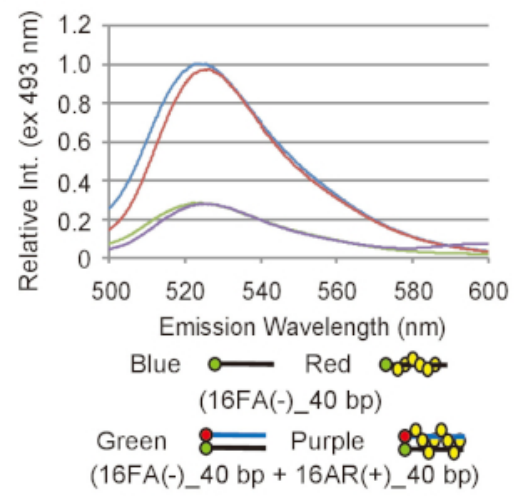

Figure 2: Measurements of the maximum FRET efficiency of the pairing and displacement assays. (A) Comparison of fluorescence spectra between the ssDNA substrate, 16FA(-), and the dsDNA product, 16FA(-) paired with 16AR(+)_40bp, of the pairing assay. Blue and red lines represent the fluorescence spectra of the substrate without and with Rad51, respectively. Green and purple lines show the fluorescence spectra of the final product without and with Rad51, respectively. (B) Comparison of fluorescence spectra between the dsDNA substrate, 16FA(-)_40bp paired with 16AR(+)_40bp, and ssDNA product, 16FA(-)_40bp, of the displacement assay. Blue and red lines represent the fluorescence spectra of the final product without and with Rad51, respectively. Green and purple lines show the fluorescence spectra of the substrate without and with Rad51, respectively. This figure is adapted from Ito et al. ${ }^{19}$ and modified. Please click here to view a larger version of this figure. 
A

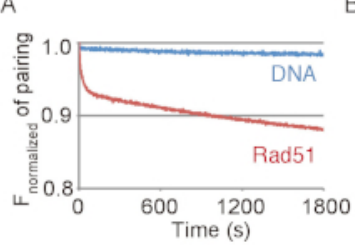

D

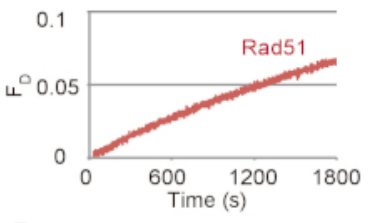

F

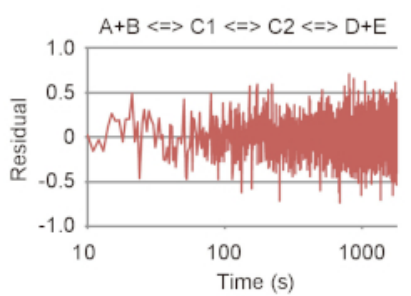

$\mathrm{H}$

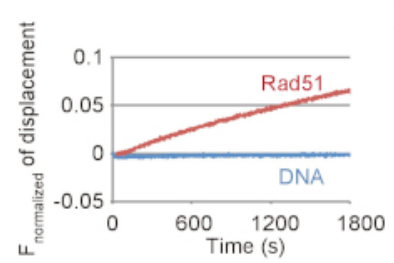

C

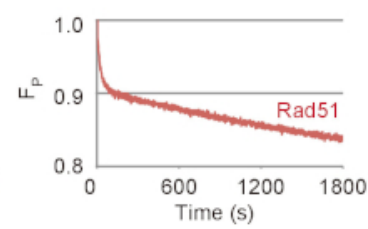

E

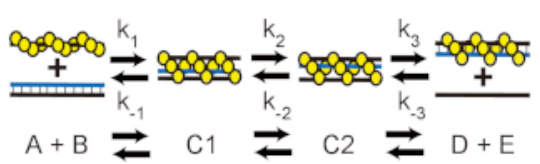

G

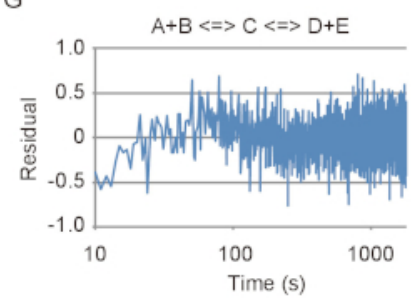

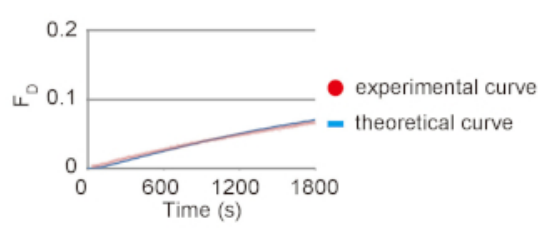

Figure 3: DNA strand pairing and displacement reactions mediated by Rad51. (A) Time course of the normalized fluorescence of the pairing reaction with or without Rad51. (B) Time course of the normalized fluorescence of the displacement reaction with or without Rad51. (C)Time course of the change in the amount of substrate in the pairing reaction with Rad51. (D) Time course of the change in the amount of substrate in the displacement reaction with Rad51. (E) A schematic of the sequential three-step reaction model. A and B correspond to the presynaptic filament and donor dsDNA. C1 corresponds to the first (immature) three-strand intermediate. C2 corresponds to the second (mature) threestrand intermediate. D and $E$ correspond to a heteroduplex and ssDNA released from C2. (F and $\mathbf{G})$ Residuals between experimental data of the DNA strand pairing assay and a theoretical curve obtained by simulation using either the three-step $(\mathbf{F})$ or two-step $(\mathbf{G})$ model. $(\mathbf{H})$ Red dots indicate experimental data from the displacement reaction with Rad51 shown in panel D. Blue line indicates the theoretical curve of the final products. The theoretical curve was generated by simulation using the reaction rate constants obtained from the pairing assay shown in panel $\mathbf{C}$. This figure is adapted from Ito et al. ${ }^{19}$ and modified. Please click here to view a larger version of this figure. 
A

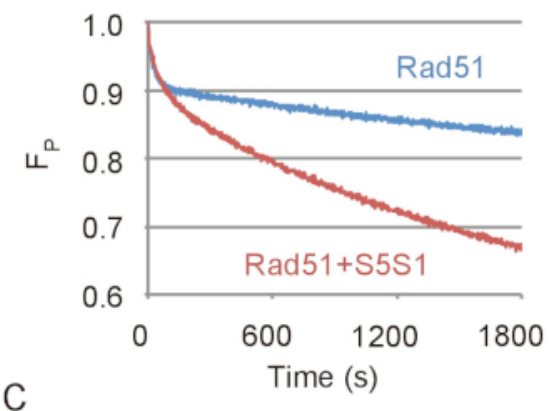

(a)

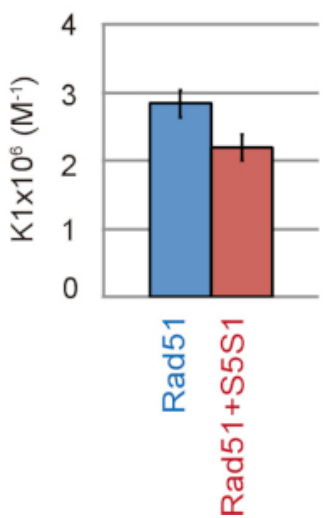

B

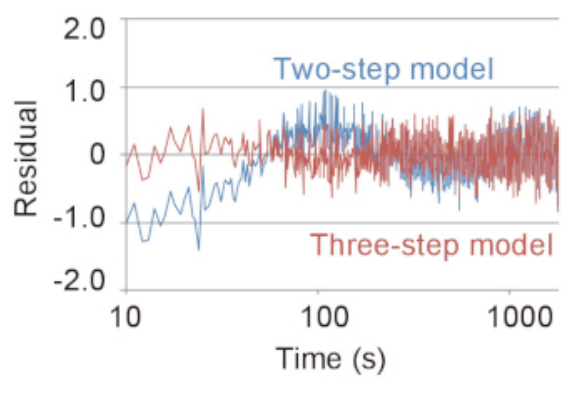

(c) (b)

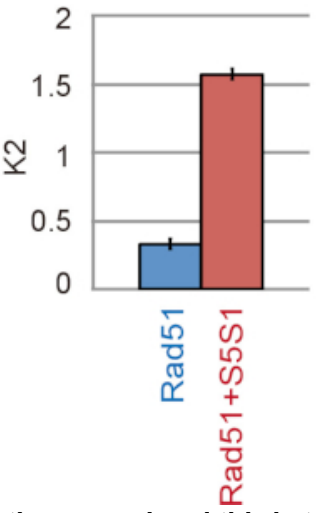

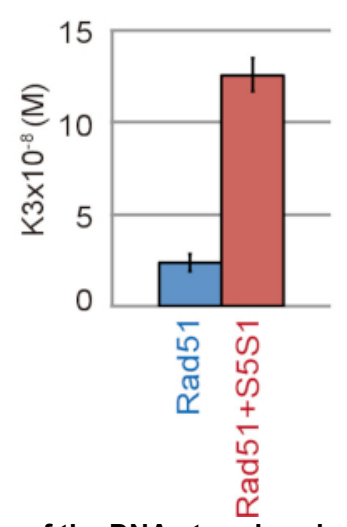

Figure 4: Swi5-Sfr1 stimulates the second and third steps of the DNA strand exchange reaction. (A) Time course of the change in the amount of substrate in the pairing reaction with or without Swi5-Sfr1 (S5S1). (B) Residuals between experimental data of the DNA strand pairing assay with Swi5-Sfr1 and a theoretical curve obtained by simulation using the two-step (blue line) or three-step (red line) model. (C) The pairing reaction shown in Figure 4A was simulated by the three-step model using the analysis program ${ }^{23}$ (see Table of Materials). The reaction equilibrium constants of each reaction step, $\mathrm{K} 1(\mathbf{a}), \mathrm{K} 2(\mathbf{b})$, and $\mathrm{K} 3(\mathbf{c})$, were obtained from the simulation. This figure is adapted from Ito et al. ${ }^{19}$ and modified. Please click here to view a larger version of this figure.

\begin{tabular}{|l|l|}
\hline \multicolumn{2}{|l|}{ Oligonucleotides for DNA strand pairing assay } \\
\hline 16FA(-) & $\begin{array}{l}\text { 5'-[FAM]-AAATGAACATAAAGTAAATAAGTATAAGGATAATACA } \\
\text { AAATAAGTAAATGAATAAACATAGAAAATAAAGTAAAGGATAT AAA -3' }\end{array}$ \\
\hline 16A(-)_40bp & 5'-AAATGAACATAAAGTAAATAAGTATAAGGATAATACAAAA-3' \\
\hline 16AR(+)_40bp & 5'-TTTTGTATTATCCTTATACTTATTTACTTTATGTTCATTT-[ROX]-3' \\
\hline Oligonucleotides for DNA strand displacement assay \\
\hline 16A(-) & $\begin{array}{l}\text { 5'-AAATGAACATAAAGTAAATAAGTATAAGGATAATACAAAATA } \\
\text { AGTAAATGAATAAACATAGAAAATAAAGTAAAGGATAT AAA -3' }\end{array}$ \\
\hline 16FA(-)_40bp & 5'-[FAM]-AAATGAACATAAAGTAAATAAGTATAAGGATAATACAAAA-3' \\
\hline 16AR(+)_40bp & 5'-TTTTTGTATTATCCTTATACTTATTTACTTTATGTTCATTT-[ROX]-3' \\
\hline
\end{tabular}

Table 1: A list of oligonucleotides used in the DNA strand pairing and displacement assays. Where applicable, the positions of fluorophores (fluorescein amidite, FAM; carboxy-x-rhodamine, ROX) are indicated in square parentheses.

\section{Discussion}

Here, we have described a detailed protocol that utilizes FRET to measure Rad51-driven DNA strand exchange in real time. Importantly, these measurements allow for the determination of reaction kinetics. While the descriptions provided above are sufficient to reproduce our published results, there are several critical points that will be described in this section. Furthermore, the advantages and disadvantages of FRET-based methodologies for studying DNA strand exchange will be discussed, along with the application of such techniques to study other aspects of DNA metabolism.

As with all biochemical reconstitutions, ensuring that all reaction substrates are of high purity is essential. It is negligent to assume the absence of contaminating activities based solely on the apparent purity of a protein preparation judged by Coomassie staining. In particular, the presence of trace amounts of nucleases or helicases can drastically affect the results of the pairing and displacement assays. Thus, we strongly encourage testing for such activities each time a new batch of protein is purified. Furthermore, it is prudent to check the purity of synthesized DNA 
substrates by native polyacrylamide gel electrophoresis. Despite many companies guaranteeing the purity of oligonucleotides, we have often found through our own testing that purity of the synthesized DNA can vary between batches.

It is important to consider the following two points when conducting experiments with quartz cuvettes. Firstly, some proteins are prone to bind quartz cuvettes nonspecifically. To counter this, BSA and polyoxyethylenesorbitan monolaurate are included in the reaction buffers. Secondly, temperature has a drastic effect on the reaction speed and fluorescence intensity. To minimize this effect, the quartz cuvette should be preincubated at $37^{\circ} \mathrm{C}$ prior to use.

Although conventional biochemical assays have been hugely useful in studying DNA strand exchange, they have several drawbacks. In a typical time-course experiment, a reaction is incubated at a certain temperature and aliquots are withdrawn at desired timepoints and deproteinized by treatment with detergent and protease to terminate the reaction. Upon completion of the time-course, samples are then subjected to electrophoresis to separate DNA substrates from products. The major advantage of the method described here is that it allows for the real-time observation of the reaction without any disturbance. Any timepoint during the reaction can be inspected without disruption to the reaction itself and there is no need to deproteinize samples or subject them to the potentially disruptive forces of electrophoresis. This is especially relevant when monitoring labile DNA structures.

Despite these strengths over conventional assays, the method described here does have some disadvantages. While the use of oligonucleotide DNA substrates for strand exchange simplifies interpretation of the results, it is important to remember that such substrates do not resemble the DNA substrates involved in HR in the cell. Some conventional assays utilize plasmid-sized DNA substrates, which are more likely to reflect the number of base-pairs that are exchanged in vivo. Furthermore, the use of topologically constrained circular dsDNA substrates in a subset of conventional assays can at least partially recreate the tensions in physiological DNA.

The application of the method described here has started to unravel the molecular mechanisms of Rad51-driven DNA strand exchange. Nonetheless, there are many interesting questions that remain to be answered. There is clear evidence that HR during meiosis requires both Rad51 and Dmc1, the meiosis-specific RecA-type recombinase in eukaryotes ${ }^{24}$. However, the lack of major biochemical differences between these two recombinases has baffled researchers in the field for years. Moreover, the roles of numerous distinct groups of recombination accessory factors has been a focal topic of research in the field of HR. In addition to elucidating the biochemical differences between Rad51 and Dmc1, we aim to investigate and compare the effects of different recombination accessory factors on the kinetics of DNA strand exchange in the immediate future. Finally, it is important to stress that the FRET-based methodology described here is not limited to the study of DNA strand exchange. With relatively minor modifications, we envision many kinds of applications for this technique in investigating functionally diverse proteins involved in DNA metabolism ${ }^{25,26,27,28}$. We hope the developments described here will provide further options to researchers belonging to many different disciplines.

\section{Disclosures}

The authors have nothing to disclose.

\section{Acknowledgments}

This work was funded by Grants-in-Aid for Scientific Research (A) (18H03985) and on Innovative Areas (15H05974) to HI, for Young Scientists (B) (17K15061) to BA, and for Scientific Research (B) (18H02371) to HT from the Japan Society for the Promotion of Science (JSPS).

\section{References}

1. Camerini-Otero, R. D., Hsieh, P. Homologous recombination proteins in prokaryotes and eukaryotes. Annual Review of Genetics. 29 (1), 509-552 (1995).

2. Cromie, G. A., Connelly, J. C., Leach, D. R. Recombination at double-strand breaks and DNA ends: conserved mechanisms from phage to humans. Molecular Cell. 8 (6), 1163-1174 (2001).

3. Pierce, A. J. et al. Double-strand breaks and tumorigenesis. Trends in cell biology. 11 (11), S52-9 (2001).

4. Haber, J. E. Genome Stability. Garland Science (2013).

5. Kowalczykowski, S. C. An Overview of the Molecular Mechanisms of Recombinational DNA Repair. Cold Spring Harbor perspectives in biology. 7 (11), a016410 (2015).

6. Bianco, P. R., Tracy, R. B., Kowalczykowski, S. C. DNA strand exchange proteins: a biochemical and physical comparison. Frontiers in bioscience: a journal and virtual library. 3, D570-603 (1998).

7. Renkawitz, J., Lademann, C. A., Jentsch, S. Mechanisms and principles of homology search during recombination. Nature Reviews Molecular Cell Biology. 15 (6), 369-383 (2014).

8. Greene, E. C. DNA Sequence Alignment during Homologous Recombination. The Journal of biological chemistry. 291 (22), $11572-11580$ (2016).

9. Sung, P., Krejci, L., Van Komen, S., Sehorn, M. G. Rad51 recombinase and recombination mediators. Journal of Biological Chemistry. 278 (44), 42729-42732 (2003).

10. Prakash, R., Zhang, Y., Feng, W., Jasin, M. Homologous recombination and human health: the roles of BRCA1, BRCA2, and associated proteins. Cold Spring Harbor perspectives in biology. 7 (4), a016600 (2015).

11. Sung, P. Function of yeast Rad52 protein as a mediator between replication protein A and the Rad51 recombinase. Journal of Biological Chemistry. 272 (45), 28194-28197 (1997).

12. Sung, P. Yeast Rad55 and Rad57 proteins form a heterodimer that functions with replication protein A to promote DNA strand exchange by Rad51 recombinase. Genes \& Development. 11 (9), 1111-1121 (1997).

13. Kurokawa, Y., Murayama, Y., Haruta-Takahashi, N., Urabe, I., Iwasaki, H. Reconstitution of DNA strand exchange mediated by Rhp51 recombinase and two mediators. PLoS biology. 6 (4), e88 (2008). 
14. Jensen, R. B., Carreira, A., Kowalczykowski, S. C. Purified human BRCA2 stimulates RAD51-mediated recombination. Nature. 467 (7316), 678-683 (2010).

15. Liu, J. et al. Rad51 paralogues Rad55-Rad57 balance the antirecombinase Srs2 in Rad51 filament formation. Nature. 479 (7372), $245-248$ (2011).

16. Lu, C.-H. et al. Swi5-Sfr1 stimulates Rad51 recombinase filament assembly by modulating Rad51 dissociation. Proceedings of the National Academy of Sciences of the United States of America. (2018).

17. Bazemore, L. R., Takahashi, M., Radding, C. M. Kinetic analysis of pairing and strand exchange catalyzed by RecA. Detection by fluorescence energy transfer. Journal of Biological Chemistry. 272 (23), 14672-14682 (1997).

18. Gupta, R. C., Bazemore, L. R., Golub, E. I., Radding, C. M. Activities of human recombination protein Rad51. Proceedings of the National Academy of Sciences. 94 (2), 463-468 (1997).

19. Ito, K., Murayama, Y., Takahashi, M., Iwasaki, H. Two three-strand intermediates are processed during Rad51-driven DNA strand exchange. Nature Structural \& Molecular Biology. 25 (1), 29-36 (2018).

20. Akamatsu, Y., Dziadkowiec, D., Ikeguchi, M., Shinagawa, H., Iwasaki, H. Two different Swi5-containing protein complexes are involved in mating-type switching and recombination repair in fission yeast. Proceedings of the National Academy of Sciences. 100 (26), 15770-15775 (2003).

21. Haruta, N. et al. The Swi5-Sfr1 complex stimulates Rhp51/Rad51- and Dmc1-mediated DNA strand exchange in vitro. Nature Structural \& Molecular Biology. 13 (9), 823-830 (2006).

22. Argunhan, B., Murayama, Y., Iwasaki, H. The differentiated and conserved roles of Swi5-Sfr1 in homologous recombination. FEBS Letters. 591 (14), 2035-2047 (2017).

23. Kuzmic, P. Program DYNAFIT for the analysis of enzyme kinetic data: application to HIV proteinase. Analytical biochemistry. 237 (2), $260-273$ (1996).

24. Brown, M. S., Bishop, D. K. DNA strand exchange and RecA homologs in meiosis. Cold Spring Harbor perspectives in biology. 7 (1), a016659 (2014)

25. Rudert, W. A. et al. Double-labeled fluorescent probes for 5' nuclease assays: purification and performance evaluation. BioTechniques. 22 (6), 1140-1145 (1997)

26. Xiao, J., Singleton, S. F. Elucidating a key intermediate in homologous DNA strand exchange: structural characterization of the RecA-triplestranded DNA complex using fluorescence resonance energy transfer. Journal of Molecular Biology. 320 (3), $529-558$ (2002).

27. Grimme, J. M. et al. Human Rad52 binds and wraps single-stranded DNA and mediates annealing via two hRad52-ssDNA complexes. Nucleic Acids Research. 38 (9), 2917-2930 (2010).

28. Algasaier, S. I. et al. DNA and Protein Requirements for Substrate Conformational Changes Necessary for Human Flap Endonuclease-1catalyzed Reaction. The Journal of biological chemistry. 291 (15), 8258-8268 (2016). 